\title{
Erfahrungen mit dem neuen Bibliothekssystem aDIS/BMS - Teil 1
}

\section{Abschalten, warten und ... hoffen: Der aDIS/BMS-Start}

Als wir, die Arbeitsgruppe zur Evaluierung von Bibliothekssystemen, in der Stadtbücherei München Ende 2007 aDIS/BMS als integriertes Bibliothekssystem mit umfassender RFID-Integration vorgestellt bekamen, hatten wir noch nicht geahnt, dass wir hier das zukünftige WLB-Bibliothekssystem vor uns sahen, und wir hatten keine konkrete Vorstellung, mit welchem großen Arbeitseinsatz die Zusammenführung und Aufbereitung unserer Bibliotheksdaten verbunden sein würde, um in die integrierte Bibliotheksdatenwelt zu wechseln. Wir waren damals nur etwas neidisch, zu sehen, wie performant und rationell die Bibliotheksbenutzung dank RFID-Technologie für Mitarbeiter und Benutzer sein kann.

Am 16.09.2013 war es aber dann auch in der WLB so weit: aDIS/BMS wurde mit unseren Daten - zunächst intern - freigeschaltet. Diesem Datum ging eine spannende zeitliche Phase voraus. Die WLB migrierte innerhalb des aDIS/BMS-Konsortiums als letzte große Bibliothek - nur kurze Zeit nach der UB Freiburg. Die Verengung des Zeitplans war für alle beteiligten Partner ein ehrgeiziges Unterfangen, insbesondere auch dadurch, dass krankheitsbedingte Ausfälle zu verkraften waren. Eine Verschiebung war jedoch nicht mehr möglich. Das BW-Projekt IBS (integriertes Bibliothekssystem in Baden-Württemberg) musste am 30.09.2013 abgeschlossen sein.

Am 04.09.2013 überschritten wir dann den „Point of no return", und unser Migrationsplan wurde "angeworfen".

Die Erstellung der Datenbasis war ein komplexes Verfahren, an dem viele Partner zeitgenau mitwirken mussten:

- Das BSZ war mit der Bereitstellung der Titelund Lokaldaten in unterschiedlichen Formaten befasst.
- Die Mitarbeiter der WLB-IT waren mit der Aufbereitung und Bereitstellung der Bibdia-, Liberound Fernleihdaten beauftragt. Weiter musste der gesamte Datenfluss geordnet ablaufen, sodass die Daten in richtiger Reihenfolge zusammengeführt werden konnten. Anpassungen im Druckerbereich mussten vorbereitet, durchgeführt und getestet werden.

- Die Projektmitarbeiterinnen der Medienbearbeitung, Ortsleihe und Fernleihe waren sehr mit der semantischen Beschreibung der Ausgangsdaten und der entsprechenden Zuordnungen im neuen System beschäftigt. Ferner mussten Schulungen durchgeführt und Geschäftsgänge neu durchdacht und ggf. angepasst werden.

- Die Firma aStec musste die Daten für das Zielsystem aufbereiten und einspielen.

- Die Informationsabteilung war mit der Erstellung von Info- und Schulungsmaterialien beschäftigt.

- Die Mitarbeiter im ZDV (Zentrum für Datenverarbeitung der Uni Tübingen) mussten zeitlich passend das Migrations-, Test-, Schulungs- und Produktionssystem bereitstellen.

Für die gesamte Aufbereitung der Daten wurde der Zeitraum vom 05.09.2013 bis 17.09.2013 benötigt. Um die Daten konsistent zu halten, wurden am 04.09.2013 die Arbeiten in Libero an den Titel-, Exemplar- und Abo-Daten eingestellt. Vom Verbund wurde anschließend in verschiedenen Formaten ein Gesamtabzug angefertigt. Dieser wurde geprüft und aStec für den aDIS/BMS-Import zur Verfügung gestellt.

Anschließend musste gewährleistet werden, dass in Bibdia keine neuen Mediennummern vergeben werden. Daher wurde die "Freitextbestellung" in Bibdia deaktiviert. Dies war die Voraussetzung für die Erstellung der Konkordanz, d.h. für die Zusammenführung der Daten aus den unterschiedlichen Systemen. Ab dem 06.09.2013 durften daher an Titel- und Exemplardaten keine Änderungen mehr 
erfolgen. Die Konkordanz Bibdia-Libero-Verbunddaten wurde nun an aStec abgeliefert.

Zum Schluss (11.09.2013) blieben noch die BibdiaBewegungsdaten übrig. Mit dem Abzug dieser Daten war dann der Ausleihstopp für unsere Benutzer verbunden.

Nun hieß es zu warten, bis alle Daten von den aStec-Mitarbeitern eingespielt wurden und zu hoffen, dass die Daten im neuen System ihren jeweils richtigen Platz finden würden.

Kompliziert war die Migration dadurch, dass die Ausgangsdaten (vor allem die Exemplardaten) über die drei Systeme Bibliotheksverbund, Bibdia und Libero verteilt waren. Verknüpfendes Element war in Bezug auf die Exemplare nur die Signatur.

Dadurch waren folgende Fälle zu unterscheiden:

1. Ein Lokalsatz mit einer Signatur trifft exakt ein Exemplar in Bibdia. Das ist ein guter Fall und kann entsprechend migriert werden.

2. Die Signatur in einem Lokalsatz trifft keine Signatur in Bibdia. Dann muss ein sogenanntes künstliches VE-Exemplar (Verbundexemplar) angelegt werden.

3. Ein Bibdia-Exemplar findet keinen Treffer im Verbund. Dann wird ein künstlicher Signaturentitel angelegt.

4. Zu einem Bibdia-Exemplar werden mehrere Titel (PPNs) im Verbund gefunden. Das ist ein Hinweis auf einen Bindeband.

5. Zu einem Titel (PPN) werden in Bibdia mehrere Signaturen gefunden. Das deutet auf einen Teiletitel hin.

6. Bei Zeitschriften trifft eine Grundsignatur mehrere Bibdia-Bände. Dann müssen Bände angelegt werden. Da hierbei eine Heuristik angewandt werden musste, und die Zuordnung von Bänden zu einem Zeitschriftentitel nicht immer eindeutig möglich war, ist die Migration im Bereich der Zeitschriftenbände „etwas durchwachsen“.

Am 16.09.2013 wurde das Ergebnis der Migration von aStec zur internen Begutachtung freigegeben. Anschließend waren Mitarbeiterinnen und Mitarbeiter der Firma aStec vor Ort, um bei den ersten Schritten zu helfen und auftretende Fragen zu beantworten.
In dieser Zeit mussten zudem die letzten Änderungen an der Benutzerdatenverwaltung und des Magazindrucks vorgenommen werden.

Der Migrationsplan hat funktioniert, und wir konnten am 17.09.2013, wie geplant, den Katalog und somit die Zugänge für die Benutzer wieder freischalten.

Als der erste Magazinzettel korrekt im Magazin gedruckt wurde, waren wir alle doch sehr erleichtert. Die Tatsache, dass wir als letzte große aDIS/BMSBibliothek migriert hatten, hat die Einführung von aDIS/BMS in der WLB sicherlich gefördert. Die Hardwarebereitstellung im ZDV hat sehr gut funktioniert. Die Maschinen arbeiteten performant, ernsthafte Ausfälle gab es bisher keine.

Dass die Systemumstellung so rund lief, ist auch den beteiligten Migrationsteams von Medienbearbeitung, Ortsleihe, Fernleihe, Information, IT und Herrn Fuchs (ehemaliger IT-Leiter der UB Tübingen) zu verdanken. Die Zusammenarbeit war äußerst konstruktiv - auch wenn es manchmal hektisch wurde. Dafür an alle auch ein großes Dankeschön.

Mit dem 17.09.2013 waren jedoch noch nicht alle Aufgaben gelöst und alle Teilaspekte abgeschlossen.

Inzwischen wurden in den Bereichen Ortsleihe / Fernleihe und Schlussstellen RFID-Antennen montiert, sodass die Vorteile der aDIS/BMS-RFID-Vollintegration - auch zur Selbstverbuchung - genutzt werden können.

\section{Wie geht es nun weiter?}

Zurzeit beschäftigen wir uns noch mit diversen kleineren und größeren Baustellen: Verbesserungen im Bereich OPAC/Benutzerkonto, Optimierung der Abläufe im Bereich Fernleihe, Überlegungen zu einer Nachmigration von Zeitschriftenbänden, mapongo-Integration in den OPAC, Statistikabfragemöglichkeiten, Verwaltung der Standortkataloge, usw.

aDIS/BMS wird im Zuge des BW-Konsortiums im Projekt aDIS-update 2014 weiterentwickelt. Dazu werden Arbeitspakete in den Bereichen Fernleihe, Druck, Signaturenregister und SAP formuliert. 
aDIS/BMS ist ein sehr komplexes System. Es wird sicherlich noch eine Zeit dauern, um in diesem System so heimisch zu werden, wie wir es in Bibdia und Libero waren. Der Start ist jedoch geschafft, der Migrationsplan konnte umgesetzt werden, und die Benutzer können unsere Dienstleistungen wieder nutzen.
Wir hoffen nun, dass es uns in der weiteren Zeit gelingt, tiefer in das Verständnis von aDIS/BMS einzutauchen, um die Geschäftsgänge und Datenverarbeitungen sowohl für Mitarbeiter als auch Benutzer weiter zu optimieren.

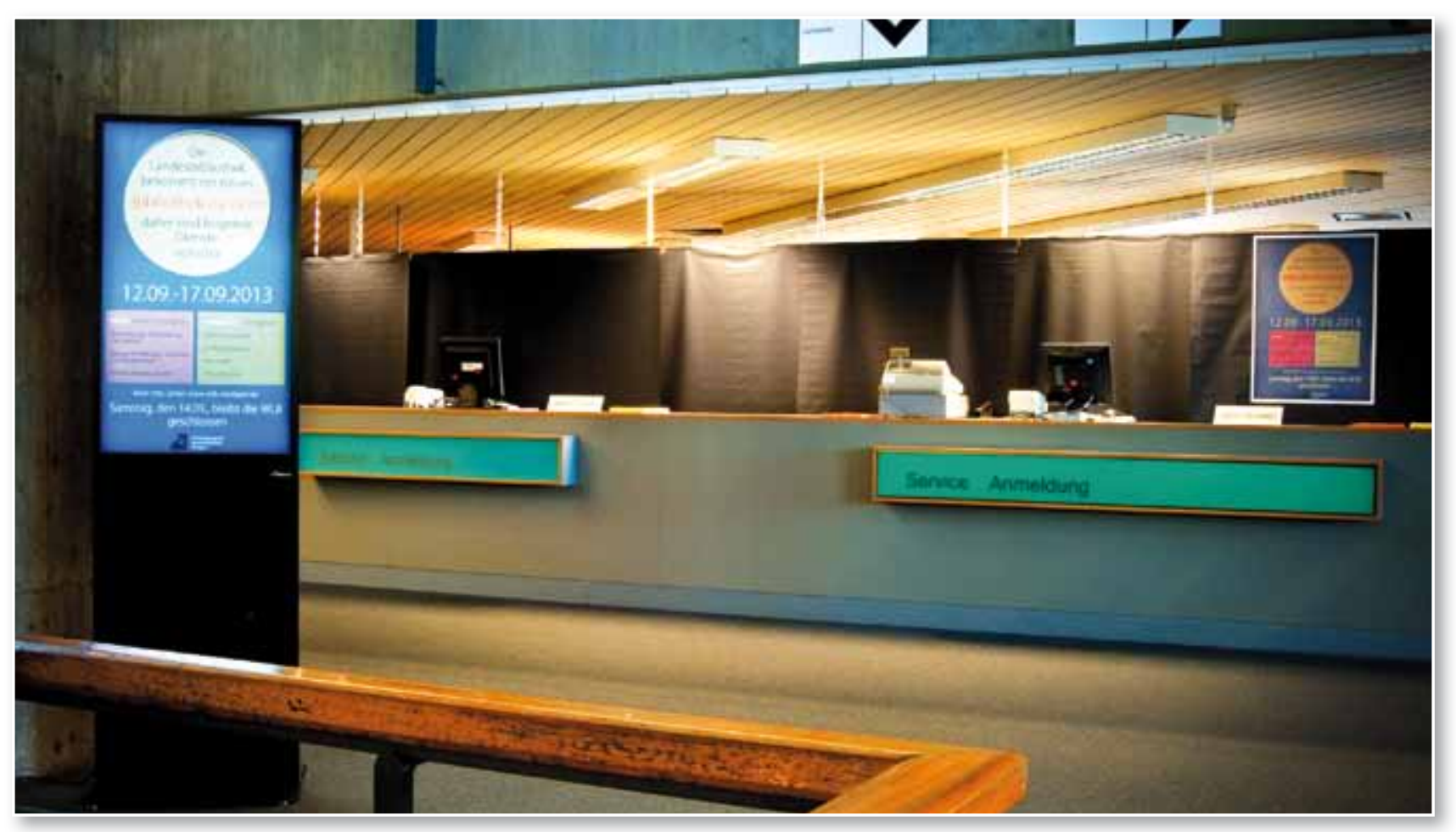

Stephan Abele

"Abschalten, warten und schulen": Die Leihstelle während der Migration

\section{Der neue Online-Katalog}

Der neue Online-Katalog bietet als Einstieg die Einfache Suche. Diese durchsucht mehrere Suchkategorien (u.a. Schlagwort, Autor, Titel) auch mit den Synonymen und Pluralformen der Suchbegriffe. Zudem kann man die Suche auf verschiedene Medienarten einschränken (Abb. 1).

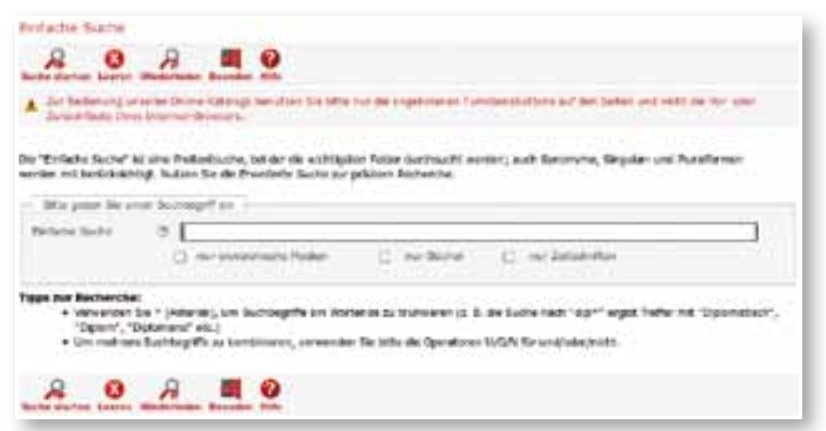

Abb. 1: Startseite Online-Katalog - Einfache Suche
Auf einer weiteren Seite steht die Erweiterte Suche mit einer Vielzahl von Sucheinschränkungen zur Verfügung. Dieses Suchformular bietet die Möglichkeit, unterschiedliche Suchkategorien zu kombinieren (Abb. 2).

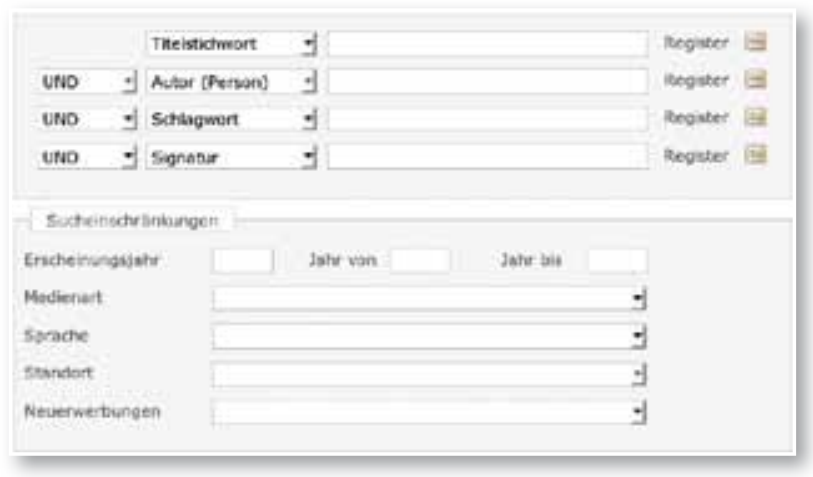

Abb. 2: Erweiterte Suche 
Vor der Systemumstellung testeten fünf Kollegen aus verschiedenen Abteilungen den neuen Katalog. Ziel war es, den Katalog an das Layout und die Gegebenheiten der Landesbibliothek anzupassen. Hierbei konnten auch erste Fehler gefunden und an die Firma aStec weitergegeben werden.

Der neue Online-Katalog bietet einige neue Funktionen und Vorteile. So kann man nach einmaliger Anmeldung mehrere Bücher bestellen. Ebenfalls sieht man direkt in der Trefferliste, ob ein Buch verfügbar ist oder nicht. Die Trefferliste kann man mit Hilfe von vorgeschlagenen Schlagwörtern, Erscheinungszeiträumen, Medienarten oder Sprachen weiter eingrenzen (Abb. 3).

\begin{tabular}{|c|c|c|}
\hline \multirow{2}{*}{$\begin{array}{l}\text { Ine fast } \\
\text { iothèque } \\
\text { Irg; suB, }\end{array}$} & \multirow[t]{2}{*}{$\otimes 2013$} & Weitere Aktionen: \\
\hline & & Meine Suche spelchern \\
\hline \multirow{3}{*}{$\begin{array}{l}\text { 1. [Red. } \\
\text { exte: }\end{array}$} & \multirow{5}{*}{$C_{2012}$} & Treffer einschränken nach: \\
\hline & & Erscheinungsjahr \\
\hline & & Schlagwort \\
\hline \multirow{2}{*}{$\begin{array}{l}\text { 12. Oktober } \\
\text { BNU; BLB. } \\
\text { jergische }\end{array}$} & & Sprache \\
\hline & & Medienart \\
\hline
\end{tabular}

Abb. 3: Auszug aus der Trefferliste

Über den Button "Meine Suchen speichern" (Abb. 3) kann man die Trefferliste abspeichern und diese beim nächsten Besuch wieder aufrufen. Sollte man aus Versehen ein falsches Medium aus dem Magazin bestellt haben, kann man dieses im Konto löschen. Dadurch steht das Medium schneller wieder für andere Benutzer zur Verfügung.

Allerdings sind noch einige Verbesserungen notwendig, u.a.:

- Suche nach weiteren Sprachen

- Suche mit der Kategorie Erscheinungsort

- weitere Einschränkungsmöglichkeiten bei Standort und Medienart

- englische Suchoberfläche

In Absprache mit den Abteilungen Information und IT-Dienste sowie der Firma aStec bemühen wir uns darum, die Verbesserungen möglichst bald durchzuführen. Besonders die Zeitschriftenbestellung bereitet derzeit noch Probleme, da bei der Migration nicht alle Jahrgangsbände sauber übernommen wurden. In der Bandanzeige fehlen zum Teil Bände, welche in der WLB vorhanden sind. Aus diesem Grund ist es wichtig, in der Kategorie Bestand zu überprüfen, ob der gesuchte Jahrgang in der WLB vorhanden ist (Abb. 4). Sollte dies der Fall sein, kann die Information bei der Bestellung des Bandes weiterhelfen. Häufig ist es möglich, den Band über die Suche nach der Signatur zu bestellen, indem man dort die Grundsignatur mit dem Jahrgang und einem Sternchen eingibt (z.B. Za 13888-1*).

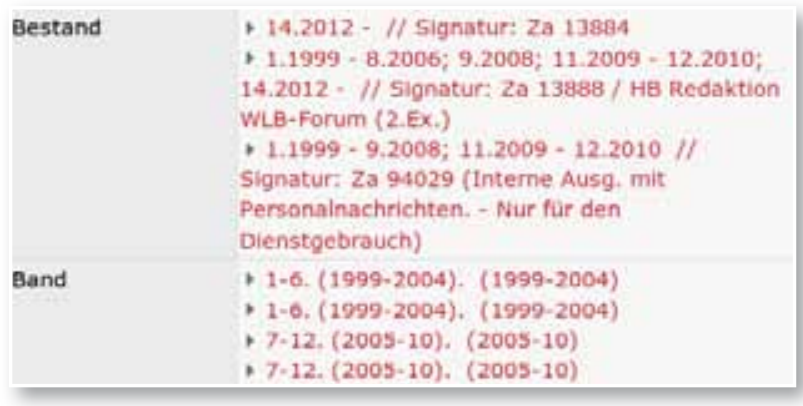

Abb. 4: Bestands- und Bandanzeige der Zeitschrift WLB-Forum

Zum neuen Katalog kommen auch viele Rückmeldungen von Benutzern. Diese Informationen helfen uns, den Katalog weiter zu verbessern. An der fehlenden Funktion "Passwort vergessen" arbeitet unsere IT-Abteilung bereits, und wir hoffen, diesen Dienst bis Mitte des Jahres wieder anbieten zu können. Ein Vorteil für Personen, welche auch die Universitätsbibliotheken im Land benutzen, ist die große Ähnlichkeit der verschiedenen Bibliothekskataloge.

Um die Umstellung auf den neuen Katalog zu erleichtern, gab es sowohl für die Benutzer als auch für die Mitarbeiter der WLB Schulungen. Rückblickend kann man sagen, dass die meisten Benutzer sich relativ schnell an den neuen Katalog gewöhnt haben. 
Auch bei der Fernleihe hat sich mit der Einführung von aDIS einiges geändert. Bisher waren Fernleihpartner in Bibdia als Benutzerkonten in einem separaten Bereich angelegt. In aDIS dagegen gibt es nun zwei verschiedene Konten pro Bibliothek. Die passive Fernleihe greift auf das Konto aus dem Bereich Körperschaften zurück. Dies ist ein Modul, das vorwiegend in der Medienbearbeitung zur Verwaltung von Lieferanten verwendet wird. Bei der aktiven Fernleihe werden Bibliotheken weiterhin als Benutzer geführt. Wie zuvor in Bibdia erhalten die Bibliotheken einige zusätzliche Angaben wie das Sigel und andere Ausleihbedingungen.

\section{Aktive Fernleihe}

\section{Bestellungen von Büchern}

Der Geschäftsgang der gebenden Fernleihe an andere Bibliotheken beginnt mit dem Ausdruck der Bestellungen und Ausheben der Bände im Magazin. Im nächsten Bearbeitungsschritt werden die Titel in der Leihstelle mit RFID-Etiketten versehen und für die Fernleihe freigebucht. In der Fernleihe werden schließlich die Bände auf die nehmenden Bibliotheken verbucht. Dazu wird die bestellende Bibliothek in aDIS aufgerufen. Mit der Verbuchung werden die Fernleihbestellnummern der nehmenden Bibliothek automatisch übernommen und auf dem Begleitschein ausgedruckt.
Doch nicht alle Bestellungen sind Routinefälle.

Bestellungen, die nicht lieferbar (z.B. entliehen) sind, oder bei denen die in der Bibliothek vorhandenen Bände nicht mit den Angaben der Bestellung übereinstimmen, müssen in anderer Weise bearbeitet werden, da sie nicht geliefert werden können. Diese werden zunächst im Zentralen Fernleihserver (ZFL) des Bibliotheksservicezentrums negativ quittiert. Danach erfolgt die Stornierung der Bestellung in aDIS. Anders stellt es sich wiederum dar, wenn die Bände vorliegen, jedoch in den Bestellungen andere Angaben eingetragen wurden. Für diese müssen zunächst in aDIS die Bestelldaten der bestellenden Bibliothek eingetragen werden. Erst im folgenden Schritt können die Bände auf die bestellende Bibliothek verbucht werden.

Mit dem neuen Bibliothekssystem werden Adressetiketten nur im DIN-A4-Format für den Versand ausgedruckt. Diese Größe zeigt sich im praktischen weiteren Ablauf als nicht optimal. Die IT-Dienste denken daher an eine Lösung, die Etiketten auf einem kleineren Format auszudrucken. Dies wäre eine wesentliche Vereinfachung für die Poststelle und den Versand.

\section{Bestellungen von Kopien und Scans}

Die Bearbeitung von Aufsatzkopien und Scans stellt sich in einer anderen Weise dar. Neu eingegangene Bestellungen können leider (noch) nicht automatisch

\begin{tabular}{|c|c|c|c|c|c|c|c|c|}
\hline & \multicolumn{7}{|c|}{ Konto bestellte Medien für } & \multirow[t]{2}{*}{ Universitätsbibliothek Fernleihe } \\
\hline & Ty & Bibl, Ausg. & Mediennummer & Signatur & bestelit & um & bis & \\
\hline$r_{1}$ & A & WLB & 27257771 & 5107321 & 250214 & 1303 & & 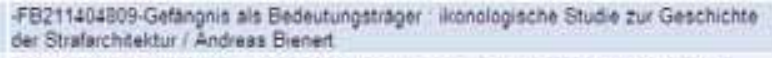 \\
\hline$\Gamma_{2}$ & A & Whe & 35132428 & $66 a / 1567$ & 28.02 .14 & 10.19 & & 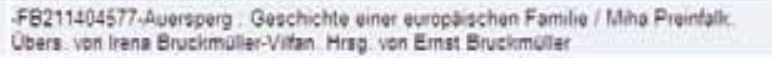 \\
\hline $\mathrm{r}_{3}$ & A & WLE & & $32 / 20924$ & 28.02 .14 & 1033 & & 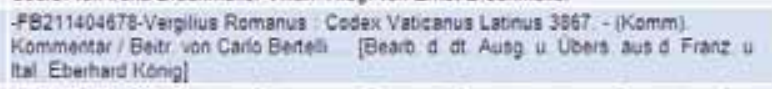 \\
\hline $\mathrm{r}_{4}$ & A & WB & 43507955 & $63 / 13818$ & 28.02 .14 & 10.42 & & $\begin{array}{l}\text { FB211404968-Rebellische Stadte vam Recht auf Stadt zur urbanen Revolution / Dand } \\
\text { Hanqy }\end{array}$ \\
\hline$\Gamma 3$ & A & WLB & 13937074 & B 85111 & 28.02 .14 & 1047 & & $\begin{array}{l}\text { Fa211404595-Japaris fertign nid chalenge polisy reform and add leaderahip / Alan } \\
\text { Ror }\end{array}$ \\
\hline$\Gamma 6$ & A & WLE & 34427279 & $432 / 90535$ & 280214 & 1221 & & 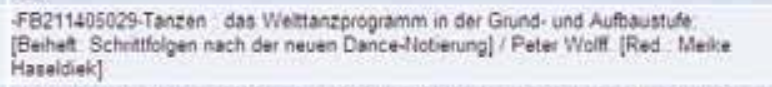 \\
\hline $\mathrm{r}_{7}$ & A & WLB & 29579068 & $40 / 6206-1$ & 200214 & 1223 & & 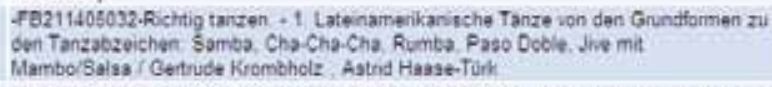 \\
\hline$\Gamma$ s & A & wLB & 35640769 & $54 / 14762.2$ & 26.02 .14 & 1225 & & 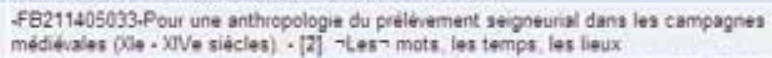 \\
\hline$r 9$ & A & WB & 36407417 & $500 / 1596$ & 200214 & 1301 & & $\begin{array}{l}\text { Fa21140504t-Zeichen und Wunder das Kino van Zhang Vimou und Wong KarWWai I } \\
\text { josul \&chuelie. Rudiger Suchsiand }\end{array}$ \\
\hline$\Gamma+10$ & A & WLB & 42979355 & $62 / 18412$ & 26.02 .14 & 1407 & & FB211405046.Pootische Gerechtigkes / hrsg. von Sebastian Donat. \\
\hline
\end{tabular}


ausgedruckt werden. Sie müssen daher im System zuerst aufgerufen und danach der Ausdruck entsprechend veranlasst werden. Von den Mitarbeitern werden im neuen Online-Katalog die Titel mit den Bandangaben überprüft und bestellt. Für Fernleihbestellungen ist ein eigenes Dienstkonto im Ausleihsystem eingerichtet. Da es sich bei der Mehrzahl der Bestellungen von Kopien und Scans um Aufsätze aus Zeitschriften handelt, ist die Überprüfung und Bearbeitung sehr zeitintensiv. Leider werden derzeit noch nicht alle Jahrgänge im Online-Katalog angezeigt, so dass häufig zunächst aufwändige Recherchen notwendig werden. In den vom Magazin gelieferten Bänden werden die bestellten Aufsätze herausgesucht und entsprechend für die Kopierstelle zum Scannen oder Kopieren gekennzeichnet. Nicht lieferbare Bestellungen müssen sowohl im Zentralen Fernleihserver (ZFL) als auch in aDIS eingetragen werden.

\section{Passive Fernleihe}

Über das Fernleihportal auf der Website recherchieren und bestellen unsere Benutzer Titel aus anderen Bibliotheken. Innerhalb des Fernleih-Portals ist für den Nutzer leider nicht erkennbar, dass der vorliegende Titel bereits selbst bestellt wurde, so dass häufig Mehrfachbestellungen auf denselben Titel entstehen, welche nachbearbeitet werden müssen.

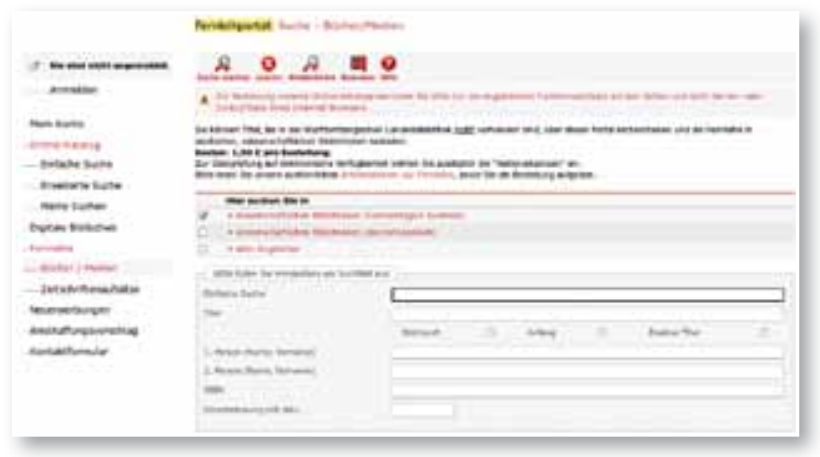

Abb. 2: Fernleihportal auf der Website der WLB

\section{Bestellungen von Büchern}

Nach Eingang der bestellten Bände in der Bibliothek werden diese in aDIS verbucht. Zuvor muss jedoch für jeden Band ein temporäres RFID-Etikett für die Selbstverbuchung mit der Fernleihbestellnummer in aDIS verknüpft werden. Diese wiederablösbaren Etiketten werden in die Bände zur weiteren Verbuchung geklebt. Mit der Rückgabe der Bände werden diese Etiketten wieder aus den Bänden entfernt.

Falls Bände geliefert werden, von denen jedoch nur Kopien bestellt wurden, müssen diese in aDIS mit Bestelltyp auf "selbständig" geändert werden. Dies ist notwendig, damit bei der Übergabeverbuchung an den Benutzer das Exemplar in aDIS nicht gelöscht wird, da bei Kopien der Vorgang an dieser Stelle als abgeschlossen gilt.

Aufwändiger gestalten sich Bestellungen, die eine Nutzungsbeschränkung für den Lesesaal haben. In aDIS müssen in diesen Fällen an drei unterschiedlichen Stellen die Vermerke für die Benutzung im Lesesaal eingetragen werden. Nur so kann gesichert werden, dass die Bände in der Leihstelle nicht als "bereitgestellt" erscheinen und möglicherweise dort von Benutzern und Mitarbeitern gesucht werden.

\section{Bestellungen von Kopien und Scans}

Eingehende Scans werden täglich automatisch ausgedruckt. Diese werden gemeinsam mit den gelieferten Kopien in aDIS verbucht, wobei der Bestelltyp „unselbständig, Kopie“ beachtet werden muss. Für die Benutzer werden Adresszettel aus dem System ausgedruckt. Auf diesen sind auch die bibliographischen Angaben des Aufsatzes vermerkt, die den Benutzern zugesandt werden.

Abschließend möchten wir uns als Mitarbeiter der Fernleihstelle sehr herzlich bei den Mitarbeitern der IT-Dienste bedanken, die uns in der Umstellungsphase auf aDIS und danach immer sehr kompetent, schnell und tatkräftig unterstützt haben. So konnten einige Anpassungen und Verbesserungen noch vorgenommen werden, die sich insgesamt auf eine schnellere Bearbeitung in den Geschäftsgängen ausgewirkt haben. Jede Systemumstellung bedeutet auch auf Mitarbeiterseite einen großen Einsatz, den wir nur gemeinsam haben bewältigen können.

Sibylle Baur 